\title{
ON THE COEFFICIENTS OF FUNCTIONS WITH BOUNDED BOUNDARY ROTATION
}

\author{
D. K. THOMAS
}

ABSTRACT. Let $V_{k}$ be the class of normalised functions of bounded boundary rotation. For $f \in V_{k}$ define

$$
M(r, f)=\max _{|z|=r}|f(z)|,
$$

and let $L(r, f)$ denote the length of $f(|z|=r)$. Then if $f(z)=z+$ $\sum_{n=2}^{\infty} a_{n} z^{n}$, it is shown that (i) $2 M(r, f)<L(r, f) \leqq k \pi M(r, f)$, and (ii) $n^{2}\left|a_{n}\right| \leqq\left(3 k / r^{n-1}\right) M\left(r, f^{\prime}\right), n \geqq 2$. The class $\Lambda_{k}$ of meromorphic functions of boundary rotation is also studied and estimates for the coefficients are given.

1. Introduction. For fixed $k \geqq 2$, let $V_{k}$ denote the class of normalised functions of boundary rotation at most $k \pi$; that is, $f \in V_{k}$ if and only if $f$ is analytic in the open unit disc $\gamma, f^{\prime}(z) \neq 0$ for $z \in \gamma, f(0)=0, f^{\prime}(0)=1$, and $f$ maps $\gamma$ onto a domain with boundary rotation at most $k \pi$. Since the boundary rotation is the total variation of the argument of the boundary tangent vector (whenever such a tangent vector exists), we have (see e.g. [5]), with $z=r e^{i \theta}$,

$$
\int_{0}^{2 \pi}\left|\operatorname{Re} \frac{\left(z f^{\prime}(z)\right)^{\prime}}{f^{\prime}(z)}\right| d \theta \leqq k \pi .
$$

$V_{2}$ is the class of normalised convex functions, and it is well known that for $2 \leqq k \leqq 4, V_{k}$ contains only univalent functions.

Suppose $f \in V_{k}$ and has Taylor expansion

$$
f(z)=z+\sum_{n=2}^{\infty} a_{n} z^{n}
$$

Then the problem $A_{n}(k)=\max \left|a_{n}\right|$ has been extensively studied, but remains largely unsolved. It is known that, for $k \geqq 2$,

$$
A_{2}(k)=k / 2, \quad A_{3}(k)=\left(k^{2}+2\right) / 6, \quad A_{1}(k)=\left(k^{3}+8 k\right) / 24,
$$

and, for $n \geqq 2$, that

$$
\left|a_{n}\right| \leqq c(k) n^{k / 2-1}
$$

Received by the editors August 5, 1971.

AMS 1970 subject classifications. Primary 30A32. 
where $c(k)$ is a constant depending only upon $k$. (4) was given in [4] with $c(k) \rightarrow \infty$ as $k \rightarrow \infty$ and in [8] with $c(k) \rightarrow 0$ as $k \rightarrow \infty$. The function $f_{0} \in V_{k}$ defined for $z \in \gamma$ by

$$
f_{0}(z)=\frac{1}{\varepsilon k}\left[\left(\frac{1+\varepsilon z}{1-\varepsilon z}\right)^{k / 2}-1\right], \quad|\varepsilon|=1,
$$

shows that equality may occur in each of (3), and also that the index of $n$ in (4) is best possible.

A class of functions closely related to $V_{k}$ is the class $\Lambda_{k}(k \geqq 2)$ of meromorphic functions defined as follows. The function $g$, given by

$$
g(z)=\frac{1}{z}+\sum_{n=0}^{\infty} b_{n} z^{n}
$$

belongs to $\Lambda_{k}$ if and only if $g$ is analytic in $\gamma^{\prime}=\gamma \backslash\{0\}, g^{\prime}(z) \neq 0$ for $z \in \gamma^{\prime}$ and $g$ maps $\gamma^{\prime}$ onto a domain with boundary rotation at most $k \pi$. For $g \in \Lambda_{k}$, we have [7], with $z=r e^{i \theta}$,

$$
\int_{0}^{2 \pi}\left|\operatorname{Re} \frac{\left(z g^{\prime}(z)\right)^{\prime}}{g^{\prime}(z)}\right| d \theta \leqq k \pi .
$$

If $g \in \Lambda_{k}$ is given by (5), then the problem $B_{n}(k)=\max \left|b_{n}\right|$ was considered in [6]. It was shown that

$$
B_{1}(k)=k / 2 \text { and } B_{2}(k)=k / 6 \text {. }
$$

It was also shown in [6] that, for $n \geqq 1,\left|b_{n}\right| \leqq C(k) n^{k / 2-3}$, for $k=2$ and $k \geqq 4$, where $C(k) \rightarrow \infty$ as $k \rightarrow \infty$.

Let $M(r, f)=\max _{|z|=r}|f(z)|$ and let $0<r<1$. The main purpose of this paper is to give estimates for the coefficients $a_{n}$ and $b_{n}$ in (2) and (5) in terms of $M\left(r, f^{\prime}\right)$ and $M\left(r, g^{\prime}\right)$ respectively. We shall also give an extremely simple proof of (4) with an improved constant $c(k)$. The methods of the paper show also that, for all $k \geqq 2$ and $n \geqq 2,\left|b_{n}\right| \leqq C(k) n^{k / 2-3}$ where $C(k) \rightarrow 0$ as $k \rightarrow \infty$. This result improves the theorem given in [6], since the estimate is now valid for all $k \geqq 2$, and since $C(k) \rightarrow 0$ as $k \rightarrow \infty$.

2. Statement of results. For $V_{k}$ we have:

THEOREM 1. Let $f \in V_{k}$ and be given by (2). Then, for any $0<r<1$,

(i) $2 M(r, f)<L(r) \leqq k \pi M(r, f)$ where $L(r)$ is the image of $\gamma_{r}=\{z:|z|=r\}$ under $f$, and

(ii) $n^{2}\left|a_{n}\right| \leqq\left(3 k / r^{n-1}\right) M\left(r, f^{\prime}\right), n \geqq 2$.

REMARK. 1. A geometrical proof of (i) was given in [2] with a worse constant. 
2. An example in [2] shows that the constant $k \pi$ in (i) is the best possible.

With the aid of this theorem we are able to prove the following corollaries:

Corollary 1. Let $f \in V_{k}$ and be given by (2). Then, for $n \geqq 5$,

$$
n\left|a_{n}\right| \leqq e^{4} / 2\left[(n / 2)^{k / 2}-1\right]
$$

For $n=2,3,4$ the inequalities (3) are better.

Corollary 2. Let $f \in V_{k}$ and be given by (2). Then, for $n \geqq 2$,

$$
n\left|a_{n}\right| \leqq 2 k e^{2}(A(1-1 / n) / \pi)^{1 / 2},
$$

where $A(r)$ is the area of $f\left(\gamma_{r}\right)$.

This last result was obtained in [1], with essentially the same constants. In [2] it was also shown that for a bounded function in $V_{k}, n a_{n}=o(1)$ as $n \rightarrow \infty$. The following extends this result.

Corollary 3. Let $f \in V_{k}$ and be given by (2). Then if the area of $f(\gamma)$ is finite, $n a_{n}=o(1)$ as $n \rightarrow \infty$, and the index of $n$ is best possible.

For $\Lambda_{k}$ we have

THEOREM 2. Let $g \in \Lambda_{k}$ and be given by (5). Then, for any $0<r<1$ and any $n \geqq 1$,

We also have

$$
n^{2}\left|b_{n}\right| \leqq\left(4 k / r^{n-1}\right) M\left(r, g^{\prime}\right) .
$$

Corollary 4. Let $g \in \Lambda_{k}$ and be given by (5). Then, for $n \geqq 2$,

$$
\left|b_{n}\right| \leqq\left(32 e^{4} k / 2^{k / 2}\right) n^{k / 2-3} .
$$

The function $g_{0} \in \Lambda_{k}$, defined by

$$
g_{0}^{\prime}(z)=-\frac{1}{z^{2}} \frac{\left(1+z^{2}-2 z(k-2) /(k+2)\right)^{(k+2) / 4}}{(1-z)^{(k-2) / 2}}
$$

shows that the index of $n$ is best possible.

3. Proofs of theorems.

Proof of TheOREM 1. (i) Write

$$
L(r)=\int_{0}^{2 \pi}\left|z f^{\prime}(z)\right| d \theta=\int_{0}^{2 \pi} z f^{\prime}(z) \exp \left[-i \arg z f^{\prime}(z)\right] d \theta
$$


Then integration by parts gives

$$
L(r)=\int_{0}^{2 \pi} f(z) \exp \left[-i \arg z f^{\prime}(z)\right] \partial_{\theta}\left(\arg z f^{\prime}(z)\right) \leqq k \pi M(r, f)
$$

on using (1). The left hand inequality is trivial.

(ii) We shall use the method of Clunie and Pommerenke [3], and shall need the following lemma.

LEMMA 1. Let $f \in V_{k}$ and, for $n \geqq 1, z=r e^{i \theta}$, put

$$
J_{n}(r)=\frac{1}{2 \pi} \int_{0}^{2 \pi}\left(z f^{\prime}(z)\right)^{\prime} z^{n+1} \exp \left[-2 i\left(\theta+\arg f^{\prime}(z)\right)\right] d \theta .
$$

Then $\left|J_{n}(r)\right| \leqq 2 k r^{n+1} M\left(r, f^{\prime}\right)$.

Proof. Let $F(z)=\left(z f^{\prime}(z)\right)^{\prime} \mid f^{\prime}(z)$. Then $(\partial / \partial \theta)\left(\theta+\arg f^{\prime}(z)\right)=\operatorname{Re} F(z)$. Integration by parts shows that

$$
J_{n}(r)=\frac{1}{\pi} \int_{0}^{2 \pi} f_{n}(z) \exp \left[-2 i\left(\theta+\arg f^{\prime}(z)\right)\right] \operatorname{Re} F(z) d \theta
$$

where

$$
f_{n}(z)=\int_{0}^{z} \zeta^{n}\left(\zeta f^{\prime}(\zeta)\right)^{\prime} d \zeta
$$

Again using integration by parts we have

and so

$$
f_{n}(z)=z^{n+1} f^{\prime}(z)-n \int_{0}^{z} \zeta^{n} f^{\prime}(\zeta) d \zeta
$$

$$
\left|f_{n}(z)\right| \leqq r^{n+1} M\left(r, f^{\prime}\right)+n M\left(r, f^{\prime}\right) \int_{0}^{r} t^{n} d t \leqq 2 r^{n+1} M\left(r, f^{\prime}\right) .
$$

From (7) we now have

$$
\left|J_{n}(r)\right| \leqq \frac{2 r^{n+1}}{\pi} M\left(r, f^{\prime}\right) \int_{0}^{2 \pi}|\operatorname{Re} F(z)| d \theta
$$

and Lemma 1 now follows on using (1).

We now prove (ii). With $\left(z f^{\prime}(z)\right)^{\prime}=f^{\prime}(z) F(z)$ we write $F(z)=2 \operatorname{Re} F(z)-$ $F(z)^{*}$, where ${ }^{*}$ denotes complex conjugate. Then with $z=r e^{i \theta}$, we have

$$
\begin{aligned}
n^{2} a_{n}= & \frac{1}{2 \pi r^{n-1}} \int_{0}^{2 \pi}\left(z f^{\prime}(z)\right)^{\prime} \exp [-i(n-1) \theta] d \theta \\
= & \frac{1}{\pi r^{n-1}} \int_{0}^{2 \pi} f^{\prime}(z) \operatorname{Re} F(z) \exp [-i(n-1) \theta] d \theta \\
& -\frac{1}{2 \pi r^{n-1}} \int_{0}^{2 \pi} f^{\prime}(z) F(z)^{*} \exp [-i(n-1) \theta] d \theta
\end{aligned}
$$


Hence

$$
\begin{aligned}
n^{2}\left|a_{n}\right| \leqq & \frac{1}{\pi r^{n-1}} \int_{0}^{2 \pi}\left|f^{\prime}(z)\right||\operatorname{Re} F(z)| d \theta \\
& \quad+\frac{1}{2 \pi r^{n-1}}\left|\int_{0}^{2 \pi} f^{\prime}(z)^{*} F(z) \exp [i(n-1) \theta] d \theta\right| \\
= & P_{1}+P_{2}, \text { say, }
\end{aligned}
$$

where in $P_{2}$ we have taken the complex conjugate.

From (1) we obtain at once $P_{1} \leqq\left(k / r^{n-1}\right) M\left(r, f^{\prime}\right)$. Now

and so

$$
f^{\prime}(z)^{*} F(z)=\left(z f^{\prime}(z)\right)^{\prime} \exp \left[-2 i \arg f^{\prime}(z)\right],
$$

$$
\begin{aligned}
P_{2} & =\frac{1}{2 \pi r^{n-1}}\left|\int_{0}^{2 \pi}\left(z f^{\prime}(z)\right)^{\prime} \exp [i(n+1) \theta] \exp \left[-2 i\left(\theta+\arg f^{\prime}(z)\right)\right] d \theta\right| \\
& =\left(1 / r^{2 n}\right)\left|J_{n}(r)\right| .
\end{aligned}
$$

Thus from Lemma $1, P_{2} \leqq\left(2 k / r^{n-1}\right) M\left(r, f^{\prime}\right)$. Hence

which proves (ii).

$$
n^{2}\left|a_{n}\right| \leqq\left(3 k / r^{n-1}\right) M\left(r, f^{\prime}\right)
$$

Proof of Corollary 1. It is well known [5] that for $f \in V_{k}$,

$$
\left|f\left(r e^{i \theta}\right)\right| \leqq \frac{1}{k}\left[\left(\frac{1+r}{1-r}\right)^{k / 2}-1\right] \text {. }
$$

With $n \geqq 5$, choose $r=1-4 / n$, then from Theorem 1 (i), with $z=r e^{i \theta}$,

$$
n\left|a_{n}\right| \leqq \frac{1}{2 \pi r^{n}} \int_{0}^{2 \pi}\left|z f^{\prime}(z)\right| d \theta \leqq \frac{1}{2 r^{n}}\left[\left(\frac{1+r}{1-r}\right)^{k / 2}-1\right]<\frac{e^{4}}{2}\left[\left(\frac{n}{2}\right)^{k / 2}-1\right] \text {. }
$$

Proof of Corollary 2. Note that

$$
\begin{aligned}
r M\left(r, f^{\prime}\right) & \leqq \sum_{n=1}^{\infty} n\left|a_{n}\right| r^{n} \\
& \leqq\left(\sum_{n=1}^{\infty} n\left|a_{n}\right|^{2} r^{n}\right)^{1 / 2}\left(\sum_{n=1}^{\infty} n r^{n}\right)^{1 / 2} \leqq\left(\frac{A(\sqrt{ } r)}{\pi}\right)^{1 / 2} /(1-r) .
\end{aligned}
$$

For $n \geqq 2$, choose $r=(1-1 / n)^{2}$, and the result follows at once from Theorem 1 (ii).

Proof of Corollary 3. This follows at once from Theorem 1 (ii) on noting that if the area of $f(\gamma)$ is finite, then $M\left(r, f^{\prime}\right)=o(1) /(1-r)$ as $r \rightarrow 1$.

The function $f_{\alpha}: f_{\alpha}(z)=(1 / \alpha)\left[1-(1-z)^{\alpha}\right]$ for $0<\alpha<1$ is convex and bounded and shows that the index of $n$ is best possible.

Proof of Theorem 2. We need a lemma analogous to Lemma 1. 
LEMMA 2. Let $g \in \Lambda_{k}$ and, for $n \geqq 2, z=r e^{i \theta}$, put

$$
K_{n}(r)=\frac{1}{2 \pi} \int_{0}^{2 \pi}\left(z g^{\prime}(z)\right)^{\prime} z^{n+1} \exp \left[-2 i\left(\theta+\arg g^{\prime}(z)\right)\right] d \theta
$$

Then $\left|K_{n}(r)\right| \leqq 3 k r^{n+1} M\left(r, g^{\prime}\right)$.

Proof. Let $G(z)=\left(z g^{\prime}(z)\right)^{\prime} / g^{\prime}(z)$. Then $G$ is analytic in $\gamma^{\prime}$ and $(\partial / \partial \theta)\left(\theta+\arg g^{\prime}(z)\right)=\operatorname{Re} G(z)$. Integration by parts shows that

$$
K_{n}(r)=\frac{1}{\pi} \int_{0}^{2 \pi} g_{n}(z) \exp \left[-2 i\left(\theta+\arg g^{\prime}(z)\right)\right] \operatorname{Re} G(z) d \theta
$$

where $g_{n}(z)=\int_{0}^{z} \zeta^{n}\left(\zeta g^{\prime}(\zeta)\right)^{\prime} d \zeta$ (Note: $n \geqq 2$ assures regularity of the integrand at $\zeta=0$.)

Again using integration by parts,

$$
g_{n}(z)=z^{n+1} g^{\prime}(z)-n \int_{0}^{z} \zeta^{n} g^{\prime}(\zeta) d \zeta,
$$

and so $\left|g_{n}(z)\right| \leqq 3 r^{n+1} M\left(r, g^{\prime}\right)$. Exactly as in Lemma 1 we now find that $\left|K_{n}(r)\right| \leqq 3 k r^{n+1} M\left(r, g^{\prime}\right)$, which proves Lemma 2 .

We now prove Theorem 2 . For $n \geqq 2$ we have

$$
\begin{aligned}
n^{2} b_{n}= & \frac{1}{2 \pi r^{n-1}} \int_{0}^{2 \pi}\left(z g^{\prime}(z)\right)^{\prime} \exp [-i(n-1) \theta] d \theta \\
= & \frac{1}{\pi r^{n-1}} \int_{0}^{2 \pi} g^{\prime}(z) \exp [-i(n-1) \theta] \operatorname{Re} G(z) d \theta \\
& -\frac{1}{2 \pi r^{n-1}} \int_{0}^{2 \pi} g^{\prime}(z) G(z)^{*} \exp [-i(n-1) \theta] d \theta
\end{aligned}
$$

Hence

$$
\begin{aligned}
n^{2}\left|b_{n}\right| \leqq & \frac{1}{\pi r^{n-1}} \int_{0}^{2 \pi}\left|g^{\prime}(z)\right||\operatorname{Re} G(z)| d \theta \\
& +\frac{1}{2 \pi r^{n-1}}\left|\int_{0}^{2 \pi} g^{\prime}(z)^{*} G(z) \exp [i(n-1) \theta] d \theta\right| \\
= & Q_{1}+Q_{2}, \text { say. }
\end{aligned}
$$

As before, $Q_{1} \leqq\left(k / r^{n-1}\right) M\left(r, g^{\prime}\right)$. Also,

$$
\begin{aligned}
Q_{2} & =\frac{1}{2 \pi r^{n-1}}\left|\int_{0}^{2 \pi}\left(z g^{\prime}(z)\right)^{\prime} \exp [i(n+1) \theta] \exp \left[-2 i\left(\theta+\arg g^{\prime}(z)\right)\right] d \theta\right| \\
& =\left(1 / r^{2 n}\right)\left|K_{n}(r)\right| \leqq\left(3 k / r^{n-1}\right) M\left(r, g^{\prime}\right)
\end{aligned}
$$


by Lemma 2 . Thus, for $n \geqq 2$,

$$
n^{2}\left|b_{n}\right| \leqq\left(4 k / r^{n-1}\right) M\left(r, g^{\prime}\right)
$$

An elementary estimate using the Cauchy integral formula shows the above estimate is also true for $n=1$, and so the proof is complete.

Proof of Corollary 4. In [6] it is shown that $g \in \Lambda_{k}$ if and only if there exists $f \in V_{k}$ with $a_{2}=0$ such that

$$
-z^{2} g^{\prime}(z)=1 / f^{\prime}(z)
$$

and that

$$
M\left(r, g^{\prime}\right) \leqq \frac{(1+r)^{k / 2+1}}{r^{2}(1-r)^{k / 2-1}}
$$

We remark that (9) is certainly not sharp, but is sufficient for our purpose. Let $n \geqq 5$ and choose $r=1-4 / n$. Then from Theorem 2 and (9) we have

$$
\left|b_{n}\right| \leqq\left(32 k e^{4} / 2^{k / 2}\right) n^{k / 2-3} \text {. }
$$

It remains only to show that this estimate is valid for $n=2,3,4$. For $n=2$, (10) follows since $\left|b_{2}\right| \leqq k / 6$. Using (8) together with the condition $a_{2}=0$, it is easily seen on equating coefficients that $\left|b_{3}\right| \leqq k^{2} / 24+k / 12$, which gives (10) for $n=3$. Similarly one can obtain $\left|b_{4}\right| \leqq k^{2} / 24+k / 20$, which again gives (10) for $n=4$.

\section{REFERENCES}

1. D. A. Brannan, On functions of bounded boundary rotation. I, Proc. Edinburgh Math. Soc. (2) 16 (1968/69), 339-347. MR 41 \#8642.

2. D. A. Brannan and W. E. Kirwan, On some classes of bounded univalent functions, J. London Math. Soc. (2) 1 (1969), 431-443. MR 40 \#4439.

3. J. Clunie and Ch. Pommerenke, On the coefficients of close-to-convex univalent functions, J. London Math. Soc. 41 (1966), 161-165. M̀R 32 \#734.

4. W. E. Kirwan, On the coefficients of functions with bounded boundary rotation, Michigan Math. J. 15 (1968), 277-282. MR 38 \#1250.

5. $\mathrm{O}$. Lehto, On the distortion of conformal mappings with bounded boundary rotation, Ann. Acad. Sci. Fenn. Ser. AI Math.-Phys. No. 124 (1952). MR 14, 743.

6. J. W. Noonan, Meromorphic functions of bounded boundary rotation, Michigan Math. J. 18 (1971), 343-352.

7. J. Pfaltzgraff and B. Pinchuk, A variational method for classes of meromorphic functions (to appear).

8. M. S. Robertson, Coefficients of functions with bounded boundary rotation, Canad. J. Math. 21 (1969), 1477-1482. MR 41 \#458.

Department of Mathematics, University of Maryland, College Park, MaryLAND 20742

Current address: Department of Pure Mathematics, University College, Swansea, Wales 